\title{
Alternation and repetition reaction times under three schedules of event sequencing '
}

ST ANLEY M. MOSS, STEPHEN ENGEL AND DOROTHY FABERMAN UNIVERSITY OF MASSACHUSETTS

Choice RTs to two equally frequent events were measured under three event alternation-repetition ratios: $3: 1,1: 1$ and 1:3. The RTs to alternating events were found to be faster than to repeating events under the $3: 1$ condition, with this effect decreasing as the degree of repetition increased. Averaged RTs under each of the conditions indicated that $S_{s}$ responded faster under the 1:1 condition, with comparable $R T s$ under the remaining two conditions. These results are discussed in terms of the subjective event probabilities under the three alternation-repetition ratios.

Bertelson (1961, 1963) and Williams (1966) have each studied the effects of event sequencing on choice reaction time (CRT) with contradictory results. In a two-CRT task, where each event occurred with equal frequency, Bertelson (1961) constructed event sequences that produced $75 \%, 50 \%$, and $25 \%$ repetitions. Under all three sequences, Bertelson found that RTs to those events which were the same as the preceding event (repetitions) were shorter than RTs to those events which differed from the preceding event (alternations). Using random sequences Williams (1966) obtained results that were the opposite of Bertelson's, i. e., repetition RTs were slower than alternation RTs. The experimental procedures used by these investigators differed. Bertelson's (1961) CRT task required Ss to rest their fingers on the response keys and to depress the appropriate finger at the onset of an event. The time between the onset of an event and the preceding response was kept well under 1 sec. Williams employed a directional response (right-left) with a central or starting position prior to each trial, along with intertrial intervals that varied between 12 and 15 sec.

The present study combined Bertelson's (1961) experimental design with a response procedure similar to that of Williams. Ss were presented with two equally probable stimuli with three ratios of alternation-repetition sequences $(3: 1,1: 1$, and $1: 3)$. One responding finger was used with an interpolated home response between trials. Intertrial intervals were considerably longer than Bertelson's (1961) but similar in length to Williams'.

Besides assessing Williams' findings under these new conditions, the present study tested the effects of uncertainties generated by the three sequences. Since the most sequential uncertainty was associated with the 1:1 condition, it was assumed that RTs under that condition would be longest. The two remaining conditions had equal amounts of sequential uncertainty, although one was basically an alternation sequence $(3: 1)$ and the other a repetition sequence $(1: 3)$.

\section{Method}

The stimulus response console had two round illuminated Dialco pushbutton keys, $1 / 2$ in. in diameter, mounted on the arm of a classroom desk-chair on which $\mathrm{S}$ rested her right arm. These keys, serving both as stimuli and responses, were $2-1 / 2$ in. from a start button at eccentric angles of $35^{\circ}$. Each key required $16 \mathrm{oz}$. operating force, with a vertical travel of 5/64 in. The lamps had a rated candle power of .55 through a white translucent cap. Halfway between, and 1 in. above the two pushbutton keys was a red-capped lamp which served as the foreperiod signal.

Stimulus sequences were programmed on punched paper tape and controlled by a BRS solid-state logic module system, which activated the foreperiod signal and one of the two stimulus lights. This system also indicated to $\mathrm{E}$ the stimulus, the response, and the time in msec. from stimulus onset to the depression of a pushbutton key.

Three random sequences of two equally probable events were constructed. Each sequence was 200 events in length. Within each block of 50 events, both events occurred with equal probability; for the three sequences, the probability of alternation equalled .75 , .50 , and .25, respectively. Events (right-left) were counterbalanced for each sequence, thus generating an additional three sequences.

The foreperiod interval, the time between onset of a $500 \mathrm{msec}$ foreperiod signal and onset of an event was $2 \mathrm{sec}$. Onset of either event activated a 1 msec $\mathrm{kHz}$ clock which was terminated when a response key was depressed. Depression of either key also turned off the event lamp. Average intertrial interval, i.e., time between onsets of successive foreperiod signals, was 12 sec which included foreperiod interval, $R T$ itself, and time required for recording the response and RT. Four blocks of 50 trials each were presented with an average of 2 min rest between blocks and a $5 \mathrm{~min}$ rest between Blocks 2 and 3 .

Ss were 30 right-handed female undergraduates from the introductory psychology courses at the University of Massachusetts randomly assigned to one of the six event sequence lists.

\section{Results and Discussion}

A check for possible position bias using median RTs indicated no significance between the right and left stimulus. A further analysis was carried out on the median RTs extracted from the data collapsed across response type (alternation, repetition) for each $S$ within each block and condition. The results of this analysis revealed no significant main effects. 


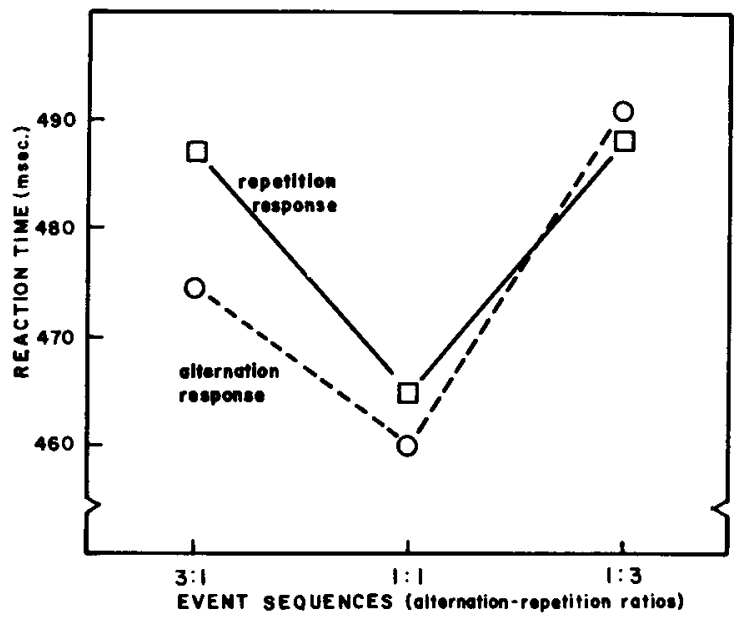

Fig. 1. Reaction times as a function of response type for the three different ratios of alternation-repetition event sequences.

Figure 1 presents the mean values for the Condition by Response type interaction ( $F=3.66, \mathrm{df}=3$ / $27, p<.05)$. Averaged across the three conditions, alternation responses are faster than repetition responses. This effect is the greatest at the 3:1 condition, less at the 1:1 condition, and slightly reversed at the 1:3 condition. This diminishing difference between the response types as the degree of sequence repetition increases, accounts for the significance of this interaction. The 1:1 condition under the present study is similar to the event sequence used by Williams. Although the differences between the RTs for the two response types are small under this condition, they are in the same direction as those obtained by Williams. A Scheffé test applied to the pairs of response types under each of the three conditions indicated that the differences were only significant under the 3:1 group $(p<.01)$.

Since there is greater sequential uncertainty associated with the 1:1 condition, it was expected to produce the slowest RT. The results of the present study indicate that contrary to expectation, these RTs were the fastest. However, the remaining two conditions, having identical amounts of sequential uncertainty, evidence slower RTs of similar magnitude.

The fallure of $\mathrm{Ss}$ to conform to the a priori expectations of $\mathrm{E}$ can perhaps be explained by Ss' application of inaccurate subjective probabilities. All Ss were questioned after the experimental session about their perception of patterns in the event structure. While complete protocols are not avallable, Ss from the 1:3 and 3:1 conditions indicated that they observed patterns and felt these to be associated with a particular event, either right or left. None of the Ss in the 1:1 group indicated awareness of event structure. Ss apparently reacted to their expectancies about event structure, assuming it was associated with a particular event rather than independent of events. It would then follow that subjective probabilities for the two events were not equal. If an $S$ were set for a particular event to occur and the other event occurred, overall RT would be longer. If Ss were apprised of the balancing of event sequencing for each event, then the increased RT generated by their subjective probabilities might disappear.

The conflicting results of Bertelson (1961) on the one hand and Williams (1966) and the present study on the other, may arise from differences in experimental procedures. Using a response procedure similar to Bertelson's, Moss (1966) suggested that unused responses can produce inhibitions leading to increased RTs. With short intertrial intervals, like Bertelson's, the persistence of inhibition by the previously unused response, as in an alternation trial, may be sufficient to delay the next response. Thus an increase in $\mathrm{RT}$ on alternation trials could represent time needed to overcome previously inhibited responses. The use of long intertrial intervals and an interpolated response may have given $S$ time to overcome response inhibition in alternation sequences.

In conclusion, the results of this study confirm the findings of Williams that alternation RTs are faster than repetition $\mathrm{RTs}$. Increasing the degree of event repetition diminishes this effect. The present data also suggested that $\mathrm{Ss}$ are misled by thelr subjective probabilities as indicated by increased RTs, when there is a high degree of alternation or repetition.

\section{References}

BERTELSON, P. Sequential redundancy and speed in a serial twochoice responding task. Quart. J. exp. Psychol, 1961, 13, 90-102.

BERTELSON, P. S-R relationships and reaction times to new versus repeated signals in a serial task. $J$. exp. Psychol., 1963, 65, 478-484. MOSS, S. Simple reaction time and response sets. Hum. Factors, 1966, 239-243.

WILLIAMS, J. Sequential effects in disjunctive reaction time: Implications for decision models. J. exp. Psychol, 1966, 71, 665-672.

Note

1. This research was in part supported by Public Health Grant MH 1104-01A1. 